\title{
Quantitative Evaluation on Heat Kernel Permutation Invariants
}

\author{
Bai Xiao ${ }^{1}$, Richard C. Wilson ${ }^{2}$, and Edwin R. Hancock ${ }^{2}$ \\ ${ }^{1}$ Department of Computer Science, University of Bath, Bath,BA2 7AF, UK \\ xb202@cs.bath.ac.uk \\ ${ }^{2}$ Department of Computer Science, University of York, York, YO10 5DD, UK \\ erh@cs.york.ac.uk
}

\begin{abstract}
The Laplacian spectrum has proved useful for pattern analysis tasks, and one of its important properties is its close relationship with the heat equation. In this paper, we first show how permutation invariants computed from the trace of the heat kernel can be used to characterize graphs for the purposes of measuring similarity and clustering. We explore three different approaches to characterize the heat kernel trace as a function of time. These are the heat kernel trace moments, heat content invariants and symmetric polynomials with Laplacian eigenvalues as inputs. We then use synthetic and real world datasets to give a quantitative evaluation of these feature invariants deduced from heat kernel analysis. We compare their performance with traditional spectrum invariants.
\end{abstract}

\section{Introduction}

The Laplacian spectrum of a graph has proved to be a convenient summary of graph structure that can be relatively easily manipulated to solve the problems such as bi-partitioning [5] and routing [1. However, the extraction of of permutation invariants for the purpose of characterising graphs has proved to be more elusive. Although the spectrum of a graph is a permutation invariant, its eigenvectors are not. To overcome this problem Luo, Wilson and Hancock 10 have used symmetric polynomials to construct a rich family of permutation invariants from the spectral matrix (the eigenvector matrix premultiplied by the square root of the eigenvalue matrix). Moreover, in prior work [12 [11 and motivated by the work of Yau [13, we have explored related invariants that can be extracted from the heat-kernel.

The invariants described above have been described in a piecemeal way and their relationship to each other has not been fully explored. Neither has their performance on large-scale object recognition tasks been fully explored. The aims in this paper are therefore twofold. First, we aim to explain the relationships between the different Laplacian invariants in a consistent and consolidated manner. Second, we aim to explore their performance on a demanding object recognition problem furnished by a large image database. 
We commence from one of the most important permutation invariants of the heat kernel, namely the trace which is found by summing and exponentiating the Laplacian eigenvalues with time. In our previous paper 12 , we showed how to characterise the shape of the heat-kernel trace using moments with respect to time. An analysis based on the Mellin transform shows that the required moment generating function is the zeta function. An alternative means of extracting invariants is to make use of heat content [6]. We show how to expand the heat content as a polynomial series in time, and demonstrate that the coefficients can be related to the eigenvalues and eigenvectors of the Laplacian. Finally, we explore the derivative of the zeta function at the origin. This is linked to the determinant of the Laplacian, and we show that it is also one of the invariants proposed by Wilson al et 10 .

The outline of this paper is as follows. In Section 2 we explain the relationship between the Laplacian eigensystem and the heat kernel trace. Section 3, we develop the three sets of invariants deduced from graph heat kernel which can be used to characterize the graphs. Section 4 offers experiments, and illustrates the utility of the different permutation invariants for graph clustering. Finally, Section 5 offers some conclusions and directions for future research.

\section{Heat Kernel on Graphs}

To commence, suppose that the graph under study is denoted by $G=(V, E)$ where $V$ is the set of nodes and $E \subseteq V \times V$ is the set of edges. Since we wish to adopt a graph spectral approach we introduce the adjacency matrix $A$ for the graph, which has elements $A(u, v)=1$ if $u, v \in E$ and 0 otherwise. We also construct the diagonal degree matrix $D$, whose elements are given by $D(u, u)=$ $\operatorname{deg}_{u}=\sum_{v \in V} A(u, v)$. From the degree matrix and the adjacency matrix we construct the Laplacian matrix $L=D-A$, i.e. the degree matrix minus the adjacency matrix. The normalized Laplacian is given by $\hat{L}=D^{-\frac{1}{2}} L D^{-\frac{1}{2}}$. The spectral decomposition of the normalized Laplacian matrix is $\hat{L}=\Phi \Lambda \Phi^{T}$, where $\Lambda=\operatorname{diag}\left(\lambda_{1}, \lambda_{2}, \ldots, \lambda_{|V|}\right)$ is the diagonal matrix with the ordered eigenvalues $\left(0=\lambda_{1}<\lambda_{2}<\ldots<\lambda_{|V|}\right)$ as elements and $\Phi=\left(\phi_{1}\left|\phi_{2}\right| \ldots \mid \phi_{|V|}\right)$ is the matrix with the corresponding ordered unit-norm eigenvectors as columns.

We are interested in the heat equation associated with the Laplacian, and this is given by $\frac{\partial h_{t}}{\partial t}=-\hat{L} h_{t}$ where $h_{t}$ is the heat kernel and $t$ is time. The heat kernel is the fundamental solution of the heat equation. It can be viewed as describing the flow of information across the edges of the graph with time. The rate of flow is determined by the Laplacian of the graph. The solution to the heat equation is $h_{t}=e^{-t \hat{L}}$. We can compute the heat kernel on a graph by exponentiating the Laplacian eigenspectrum(see e.g. Chung [2]), i.e. $h_{t}=\Phi \exp [-\Lambda t] \Phi^{T}=\sum_{i=1}^{|V|} \exp \left[-\lambda_{i} t\right] \phi_{i} \phi_{i}^{T}$. The heat kernel is a $|V| \times|V|$ matrix, and for the nodes $u$ and $v$ of the graph $G$ the resulting element is

$$
h_{t}(u, v)=\sum_{i=1}^{|V|} \exp \left[-\lambda_{i} t\right] \phi_{i}(u) \phi_{i}(v)
$$




\section{$3 \quad$ Heat Kernel Invariants}

In this section, we will describe a number of invariants that can be computed from the heat kernel matrix of the graph. These include the zeta function, heat content invariants, and the unary attributes from symmetric polynomials.

\subsection{Zeta Function and Heat Kernel Trace Moments}

The trace of the heat kernel is the sum of the diagonal elements of the heat kernel matrix of the graph.

$$
Z(t)=\operatorname{Tr}\left[h_{t}\right]=\sum_{i=1}^{|V|} \exp \left[-\lambda_{i} t\right]
$$

The purpose in this part is to use the shape of the heat kernel trace function as a means of characterizing graph structure. Our characterization is found by taking moments of trace function over time.

To commence our development, we consider the zeta function associated with the Laplacian eigenvalues. The zeta function is defined by $\zeta(s)=\sum_{\lambda_{i} \neq 0} \lambda_{i}^{-s}$. In other words, it is the result of exponentiating and summing the reciprocal of the non-zero Laplacian eigenvalues.

To establish the link between the zeta function and the trace of the heat kernel we make use of the Mellin transform. The Mellin Transform of the function $f(t)$ is $w(s)=\int_{0}^{\infty} t^{s-1} f(t) d t$. Hence, the heat kernel trace has Mellin transform

$$
\int_{0}^{\infty} \operatorname{Tr}\left[h_{t}\right] t^{s-1} d t=\int_{0}^{\infty} \sum_{i=1}^{|V|} e^{-\lambda_{i} t} t^{s-1} d t
$$

Suppose that the zero eigenvalue $\lambda_{1}=0$ has multiplicity $C$. Removing this eigenvalue from the sum under the integral, we have $\int_{0}^{\infty} \operatorname{Tr}\left[h_{t}\right] t^{s-1} d t=\int_{0}^{\infty}[C+$ $\left.\sum_{\lambda_{i} \neq 0} e^{-\lambda_{i} t}\right] t^{s-1} d t$. To develop this expression for the heat kernel trace, we note that the gamma function $\Gamma(s)$ is defined to be $\Gamma(s)=\int_{0}^{\infty} t^{s-1} e^{-t} d t$. As a result $\int_{0}^{\infty} t^{s-1} e^{-\lambda_{i} t} d t=\frac{\Gamma(s)}{\left(-\lambda_{i}\right)^{s}}$. Hence, we can write $\int_{0}^{\infty}\left(\operatorname{Tr}\left[h_{t}\right]-C\right) t^{s-1} d t=$ $\sum_{\lambda_{i} \neq 0} \frac{\Gamma(s)}{\left(-\lambda_{i}\right)^{s}}$. So finally, the zeta-function is given by $\zeta(s)=\sum_{\lambda_{i} \neq 0}\left(-\lambda_{i}\right)^{-s}=$ $\frac{1}{\Gamma(s)} \int_{0}^{\infty}\left(\operatorname{Tr}\left[h_{t}\right]-C\right) t^{s-1} d t$. In other words, the zeta function is related to the Mellin moments of the heat kernel trace. It is hence a way to characterizing the shape of the heat kernel trace with time. In our experiments, we take zeta functions with different integer input to construct the feature vector.

\subsection{Heat Content Invariants}

We now turn our attention to heat content invariants. In a recent paper McDonald and Meyers [6] have described a set of differential invariants that can be 
derived from the heat content of the heat kernel. The heat content is the sum of the entries of the heat kernel over the nodes of the graph and is given by

$$
Q(t)=\sum_{u \in V} \sum_{v \in V} \sum_{k=1}^{|V|} \exp \left[-\lambda_{k} t\right] \phi_{k}(u) \phi_{k}(v)
$$

McDonald and Meyers [6] have shown that the heat content can be expanded as a polynomial in time, i.e. $Q(t)=\sum_{m=0}^{\infty} q_{m} t^{m}$. We expand $\exp \left[-\lambda_{k} t\right]$ by using MacLaurin series and substitute it with Equation 4, we have $Q(t)=$ $\sum_{m=0}^{\infty} \sum_{u \in V} \sum_{v \in V} \sum_{k=1}^{|V|} \phi_{k}(u) \phi_{k}(v) \frac{\left(-\lambda_{k}\right)^{m} t^{m}}{m !}$. As a result the coefficients $q_{m}$ appearing in the polynomial expansion of $Q(t)$ are given by

$$
q_{m}=\sum_{k=1}^{|V|}\left\{\left(\sum_{u \in V} \phi_{k}(u)\right)^{2}\right\} \frac{\left(-\lambda_{k}\right)^{m}}{m !}
$$

As a result, the time-series coefficient $q_{m}$ does not depend on the permutation order of the nodes of the graph.

The time-series coefficients $q_{m}$ are a set of invariants which can be used for graph characterization. Since they depend on both eigenvalues and eigenvectors, they are more likely to be unique than invariants purely based in eigenvalues which are likely to be affected by problems of co-spectrality 2 . We will explore the use of the time-series polynomial coefficients for the purpose of graph clustering. To do this we construct a feature vector $\vec{B}=\left(q_{1}, q_{2}, \ldots, q_{k}\right)^{T}$ from the $k$ leading coefficients of the heat content polynomial.

\subsection{Unary Attributes with Symmetric Polynomials}

In this part, we illustrate the relationship between the derivative of the zeta function at the origin and the symmetric polynomial invariants of Wilson, Hancock and Luo 10 .

In their recent paper Wilson, Hancock and Luo [10 have reported a family of permutation invariants that can be computed by applying symmetric polynomials $S_{r}$, to the elements from the graph spectral matrix $\sqrt{\Lambda} \Phi$.

There are of course alternative invariants that can be computed from the spectrum of the Laplacian. The most obvious of these is the sum of eigenvalues or the trace of the normalized Laplacian eigenvalue matrix of the graph. Then if we use the eigenvalues of the normalized Laplacian matrix as input for the symmetric polynomial $S_{r}$, the first symmetric polynomial is also the trace of the normalized Laplacian and the last, which is $S_{1}\left(\lambda_{1}, \lambda_{2}, \ldots, \lambda_{n}\right)=\prod_{i=1}^{n} \lambda_{i}$, is related to the derivative of the zeta function at the origin which is $\zeta^{\prime}(0)=\sum_{\lambda_{i} \neq 0}\left\{-\ln \lambda_{i}\right\}=$ $\ln \left\{\prod_{\lambda_{i} \neq 0}\left(\frac{1}{\lambda_{i}}\right)\right\}$. Moreover, in the paper [10, Wilson, Hancock and Luo found it necessary to perform logarithmic squashing of the polynomials to bring them into a convenient dynamic range. This property arises naturally through the analysis of the zeta function. 
In our experiments, we will use the symmetric polynomial to construct unary attributes for graph characterization. The input for the symmetric polynomial are the columns of the spectral matrix and the eigenvalues of the normalized Laplacian matrix.

\section{Experiment}

In this section, we will apply our methods to synthetic and real world image databases. We first use synthetic data to quantitatively investigate heat kernel invariants behaviors. This study is divided into two parts. We commence by comparing the three new methods as ways for graph characterization, and then evaluate the stability of them. In the second experiment, we will apply our methods to shape analysis. Here we use a shock tree database which composed of 150 silhouettes of 10 different types of object 9 .

\subsection{Graph Characterization}

In the first experiment, we test our methods for graph characterization on synthetic graphs. To this end, we generate five different seed graphs with different structure, i.e. number of nodes and number of edges. We then apply random edit operations to the five seed graphs to simulate the effects of noise. The edit operations are edge deletion, edge insertion and node deletion. These operations are used to generate five structurally related graph clusters, which we would hope to separate using our methods. In Figure 1, we show the original seed graphs. For each seed graph, we randomly perform 3 to 10 edit operations to obtain 70 graph variants.
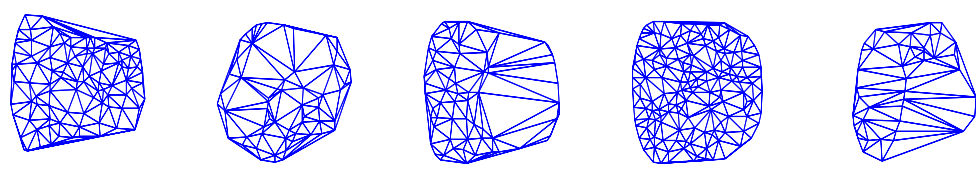

Fig. 1. Example of the seed graphs

We commence by illustrating the behavior of the zeta function. ¿From leftto-right and top-to-bottom in Figure 2 we show the values of $\zeta(1), \zeta(2), \zeta(3)$ and $\zeta(4)$ for the 70 randomizations of each seed graph. In each plot the different curves are for the different seed graphs, and the $\mathrm{x}$-axis is the index number of the randomization. The main feature to note is that the curves for the different graph clusters are well separated, and that the individual values of the zeta function do not vary significantly with randomization index. When $s=1$ and $s=2$, the fluctuations of curves $\zeta(s)$ are generally small. However, the fluctuation is larger when $s=4$. This is because when $s$ becomes large the small eigenvalues will play a dominant role in the $\zeta$ function. 

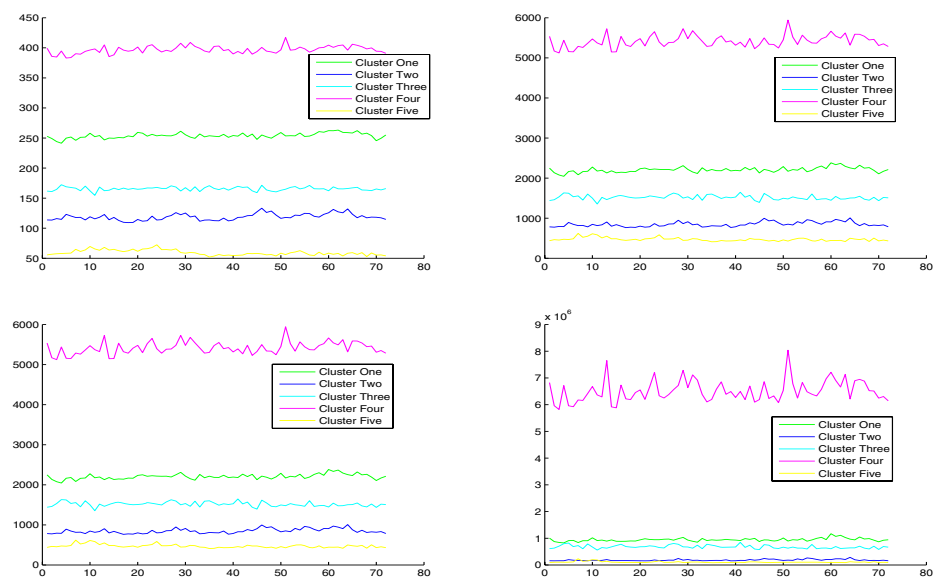

Fig. 2. Zeta function $\zeta(s)$ for randomizations of the five seed graphs. From left to right, and top to bottom, $s=1,2,3$ and 4 respectively.
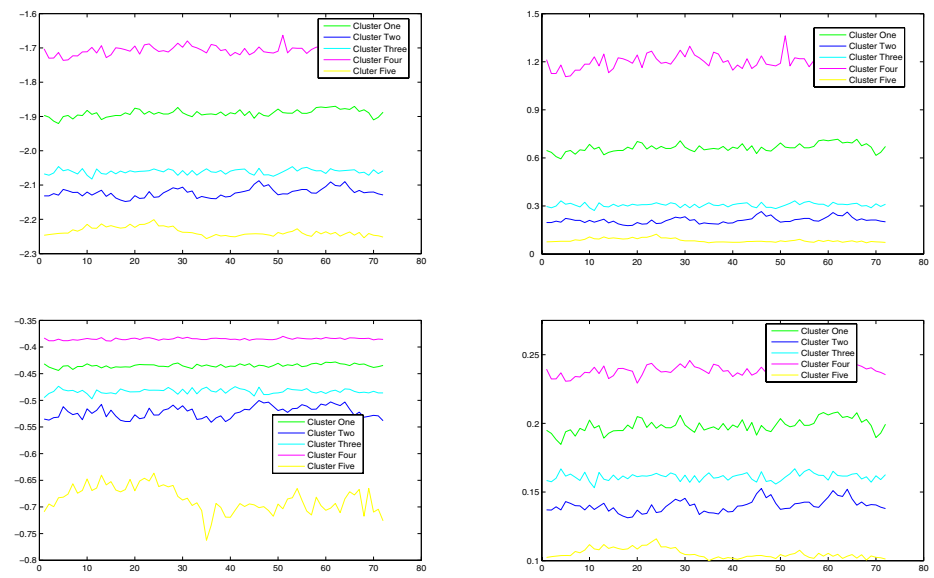

Fig. 3. Individual heat content invariants $q_{n}$ for five cluster random graphs(from left to right, and top to bottom, $n=1,2,3$ and 4 respectively).

In Figure 3, we plot the four leading heat content invariants coefficients $q_{1}, q_{2}, q_{3}$ and $q_{4}$ separately as a function of the randomization index for the five seed graphs. The coefficients are relatively stable under randomized edit operations. The curves are well separated for the different objects, and the fluctuations are relatively small.

We turn our attention to the unary features from symmetric polynomials. In the left of Figure 4, we plot the mean values of the symmetric polynomials for the five graph clusters, as a function of polynomial order. Here the arguments of the polynomials are the elements of the spectral matrix $\sqrt{\Lambda} \Phi$. The different curves in 

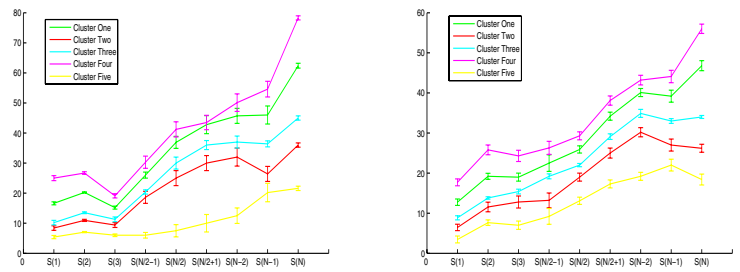

Fig. 4. Results for symmetric polynomials using spectral matrix elements for five cluster random graphs(left) and symmetric polynomials using normalized Laplacian eigenvalues(left) for five cluster random graphs

the plot are for the different seed graphs. We select 9 different symmetric polynomials for investigation. These are the first three symmetric polynomials $S_{1}, S_{2}, S_{3}$, the middle three symmetric polynomials $S_{f i x\left(\frac{N}{2}\right)-1}, S_{f i x\left(\frac{N}{2}\right)}, S_{f i x\left(\frac{N}{2}\right)+1}$ and last three symmetric polynomials $S_{N-2}, S_{N-1}, S_{N}$, where $N$ is the number of node in the graph. The error bars show the standard deviation over the different sets of graphs of the same cluster. The right plot of Figure 4 repeats the analysis using the normalized Laplacian spectrum as the arguments of the polynomials. It is clear that the first three and last three symmetric polynomials computed using the normalized Laplacian eigenvalues and spectral matrix elements as inputs can be used for graph clustering. We also get a better separation of objects when using the polynomials from the normalized Laplacian spectrum rather than the elements of the spectral matrix.

\subsection{Stability Evaluation}

We now evaluate the stability of different graph characterization methods. We study five different methods. These are a) heat content invariants, b) the zeta function values, c) symmetric polynomials with Laplacian eigenvalues as arguments, d) symmetric polynomials with spectral matrix elements as arguments and e) the Laplacian spectrum. We generate a random graph with thirty five vertices and one hundred and twenty eight edges. We compute the Euclidean distance between the coordinate vector from the principal component analysis (PCA) projection of the graph feature vector and its modified variant obtained by deleting between 3 and 27 edges from the random graph. The edge to be deleted is chosen at random. For each deletion, we perform 30 trails in order to obtain the average distance together with its standard deviation.

In Figure 5, the x-axis in each plot shows the number of deleted edges, while the $y$-axis shows the mean Euclidean distance between the original random graph and the modified variant graph together with the standard deviation. These plots show that the five characterizations we proposed in this chapter have an approximately linear relationship between the Euclidean distance and the number of 

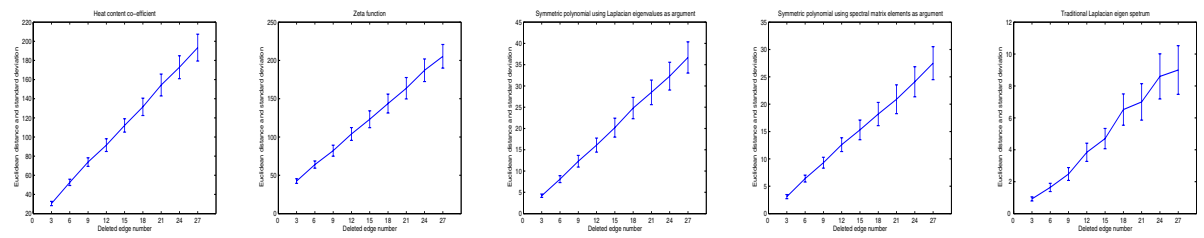

Fig. 5. Euclidean distance and standard deviation of the random graph experiments

deleted edges. The traditional characterization based on the Laplacian spectrum seems marginally less linear.

\subsection{Applications on Shape Analysis}

We have also explored the application of our new graph characterization methods to shape analysis. Here graph based methods have provided a powerful means of characterizing the underlying variations in shape due to deformation, articulation or natural class variability 7 . We have applied our methods to shock trees. This is a tree structure based representation of the differential structure of the boundary of a $2 \mathrm{D}$ shape. It is obtained by locating the shape skeleton, and examining the differential behavior of the radius of the bitangent circle from the skeleton to the object boundary, as the skeleton is traversed [8]. Recently, the shock tree extraction process has been further improved by Torsello and Hancock 9 .

In this paper we use these methods in [9] to extract a tree representations from the silhouettes of $2 \mathrm{D}$ shape. A representative view of each object studied is shown in Figure 6 .

In the database, there are over 15 shapes from 10 classes. For each shape we can construct a tree representation of the skeleton. We compute the different invariants described earlier. A GMM(Gaussian Mixture Model) 3 supervised learning process is applied to the feature vectors constructed from the invariants for the purposes of classification. In Figure [7, we plot the proportion of shapes from the database correctly classified as the number of shape classes is increased.
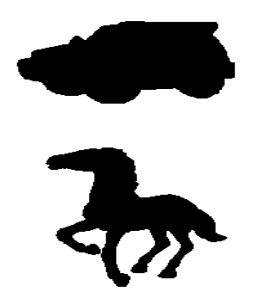
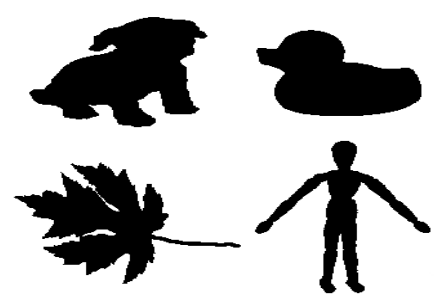
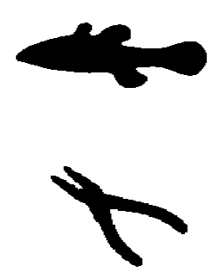

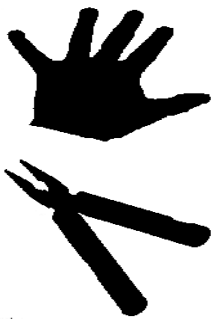

Fig. 6. Sample views of shape silhouettes 


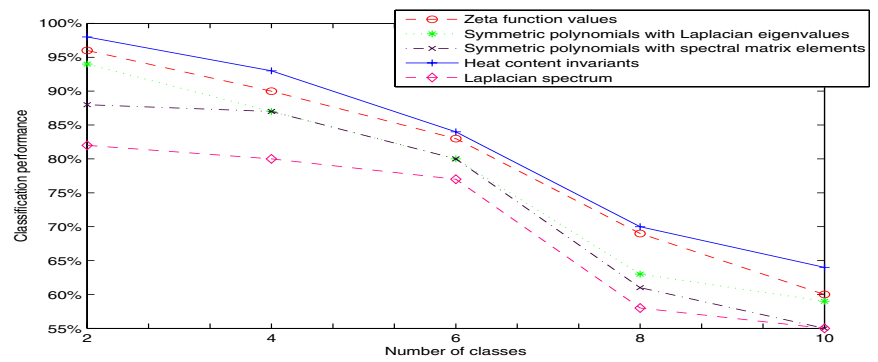

Fig. 7. Shape silhouettes classification performance as a function of silhouette numbers

In this experiment, we choose 5 shapes from each class as training data to generate the object classifier. With more classes been inserted, the classifier contains more trained Gaussian classes. All five methods explored decreases in classification accuracy as the size of the database increases. The best performance is given by the heat-content invariants, closely followed by the feature vector of zeta-function values. The poorest performance is given by the spectrum of Laplacian eigenvalues.

\section{Conclusion}

In this paper we have explored the use of several invariants computed from the heat kernel to characterize graphs. We have experimented with each of the newly proposed graph characterizations on synthetic and real world databases. The new methods show advantages over traditional methods in our experiments.

There are a number of ways in which the work reported in this paper can be extended. First, we aim to explore in more depth the links with spectral geometry, and the use of differential invariants computed from the spectrum of the Laplace-Beltrami operator [4. One interesting route here may be to explore the use of the Gauss-Bonnet theorem or Atiyah-Singer index theorem to compute topological invariants. A second line of investigation will be to explore in more depth the link between the heat kernel invariants and symmetric polynomials. Finally, we intend to explore different application domains. For instance, we will explore whether the invariants described in this paper can be used to characterize molecular structures and small world networks.

\section{References}

1. Atkins, J.E., Bowman, E.G., Hendrickson, B.: A spectral algorithm for seriation and the consecutive ones problems. SIAM J. Comput. 28, 297-310 (1998)

2. Chung, F.R.K.: Spectral graph theory. American Mathematical Society (1997)

3. Figueiredo, M.A.F., Jain, A.K.: Unsupervised learning of finite mixture models. IEEE Trans. PAMI 24(3), 381-396 (2002)

4. Chaval, I., Farkas, H.M.: Extremal kahler metrics ii. Differential Geometry and Complex Analysis, 95-114 (1985) 
5. Shi, J., Malik, J.: Normalized cuts and image segmentation. IEEE Trans. PAMI 22, 888-905 (2000)

6. McDonald, P., Meyers, R.: Diffusions on graphs, poisson problems and spectral geometry. Transactions on American Mathematical Society 354, 5111-5136 (2002)

7. Mori, G., Belongie, S.J., Malik, J.: Efficient shape matching using shape contexts. IEEE Trans. Pattern Anal. Mach. Intell. 27(11), 1832-1837 (2005)

8. Sebastian, T.B., Klein, P.N., Kimia, B.B.: Recognition of shapes by editing their shock graphs. IEEE Trans. Pattern Anal. Mach. Intell. 26(5), 550-571 (2004)

9. Torsello, A., Robles-Kelly, A., Hancock, E.R.: Discovering shape classes using tree edit-distance and pairwise clustering. International Journal on Computer Vision 72(3), 259-285 (2007)

10. Wilson, R.C., Luo, B., Hancock, E.R.: Pattern vectors from algebraic graph theory. IEEE Trans. PAMI 27, 2220-2237 (2003)

11. Xiao, B., Hancock, E.R.: Clustering shapes using heat content invariants. In: ICIP (1), pp. 1169-1172 (2005)

12. Xiao, B., Hancock, E.R.: Trace formula analysis of graphs. In: SSPR/SPR, pp. 306-313 (2006)

13. Yau, S.T., Schoen, R.M.: Differential geometry. Science Publication (1988) 